\title{
Transverse Depinning in Strongly Driven Vortex Lattices with Disorder
}

\author{
C.J. Olson and C. Reichhardt \\ Department of Physics, University of California, Davis, CA 95616
}

(November 11, 2017)

\begin{abstract}
Using numerical simulations we investigate the transverse depinning of moving vortex lattices interacting with random disorder. We observe a finite transverse depinning barrier for vortex lattices that are driven with high longitudinal drives, when the vortex lattice is defect free and moving in correlated 1D channels. The transverse barrier is reduced as the longitudinal drive is decreased and defects appear in the vortex lattice, and the barrier disappears in the plastic flow regime. At the transverse depinning transition, the vortex lattice moves in a staircase pattern with a clear transverse narrow-band voltage noise signature.
\end{abstract}

PACS numbers: $74.60 . \mathrm{Ge}$

The dynamics of driven vortex lattices interacting with disorder exhibit a wide variety of interesting nonequilibrium behavior and dynamic phase transitions. Experiments [1 8], simulations [9 14], and theory [9 15 18] suggest that at low drives the vortex lattice is disordered and exhibits plastic or random flow while at higher drives the lattice can undergo a reordering transition and flow elastically. In this highly driven state it was suggested by Koshelev and Vinokur [9] that the flux lattice forms a moving crystal. In subsequent theoretical work, Giamarchi and Le Doussal [15,16] proposed that the reordered state is actually an ordered moving glass phase and that the vortices travel in highly correlated static channels. In other work [17]18, it has been proposed that these channels may be decoupled, producing a smectic structure. Simulations [10, 11, 13, 14 and experiments [7] have found evidence for both smectic as well as more ordered moving vortex lattice structures.

A particularly intriguing prediction of the theory of Giamarchi and Le Doussal is that, in the highly driven phase, the moving lattice has a diverging potential barrier against a transverse driving force, resulting in the existence of a finite transverse critical current. This transverse critical current has been observed in simulations by Moon et al. [10] and Ryu et al. 11] in the highly driven phase. Large transverse barriers have also been seen in systems containing periodic pinning [19]. Also, recent experiments [8] involving STM images of moving vortices reveal that the vortex lattice moves along one of its principle axes rather than in the direction of the drive (as predicted by [20]), suggesting that the moving lattice is stable to a small transverse force component.

Although the existence of a transverse critical current has been confirmed in simulations, there has been no numerical study of the properties of the critical current, such as the dependence of the barrier size on the strength of the longitudinal drive or on the defectiveness of the vortex lattice. It would also be very interesting to understand the dynamics of the vortices at the transverse depinning transition, and relate this to experimental mea- sures such as voltage noise spectra.

In this work we report a simulation study of the transverse depinning transition in driven vortex lattices interacting with random disorder. We find that at high longitudinal drives, when the vortex lattice is defect free and moves in correlated 1D channels along one of its principle axes, a finite transverse depinning barrier is present. For lower drives the transverse barrier is reduced but still present, even in the decoupled channel limit when adjacent channels slip past each other and some defects in the vortex lattice appear. For the lowest drives the flux lattice becomes highly defected as it enters the plastic flow phase, and the transverse barrier is lost. In the high driving limit at the transverse depinning transition, the vortex lattice spends most of its time moving along the longitudinal direction, but periodically jumps in the transverse direction by one lattice constant. The vortex lattice can thus be seen to move in a staircase like fashion, keeping its principle axis aligned in the original direction of longitudinal driving. As the transverse force is increased the frequency of the jumps in the transverse direction increases. This motion produces a clear washboard signal in the transverse velocity which can be detected for transverse drives up to ten times the transverse depinning threshold.

We consider a 2D slice of a system of superconducting vortices interacting with a random pinning background. The applied magnetic field $\mathbf{H}=H \hat{\mathbf{z}}$ is perpendicular to our sample, and we use periodic boundary conditions in $x$ and $y$. The $T=0$ overdamped equation of motion for a vortex is:

$$
\mathbf{f}_{i}=\eta \mathbf{v}_{i}=\mathbf{f}_{i}^{v v}+\mathbf{f}_{i}^{v p}+\mathbf{f}_{d}+\mathbf{f}_{i}^{T},
$$

where $\mathbf{f}_{i}$ is the total force acting on vortex $i, \mathbf{v}_{i}$ is the velocity of vortex $i$, and $\eta$ is the damping coefficient, which is set to 1 . The repulsive vortex-vortex interaction is given by $\mathbf{f}_{i}^{v v}=\sum_{j=1}^{N_{v}} A_{v} f_{0} K_{1}\left(\left|\mathbf{r}_{i}-\mathbf{r}_{j}\right| / \lambda\right) \hat{\mathbf{r}}_{i j}$ where $\mathbf{r}_{i}$ is the position of vortex $i, \lambda$ is the penetration depth, $f_{0}=\Phi_{0}^{2} / 8 \pi \lambda^{3}$, the prefactor $A_{v}$ is set to 3 [13], and $K_{1}(r / \lambda)$ is a modified Bessel function which 
falls off exponentially for $r>\lambda$, allowing a cutoff in the interactions to be placed at $r=6 \lambda$ for computational efficiency. We use a vortex density of $n_{v}=0.75 / \lambda^{2}$ giving the number of vortices $N_{v}=864$ for a sample of size $36 \lambda \times 36 \lambda$. The pinning is modeled as randomly placed attractive parabolic traps of radius $r_{p}=0.3 \lambda$ with $\mathbf{f}_{i}^{v p}=\left(f_{p} / r_{p}\right)\left(\left|\mathbf{r}_{i}-\mathbf{r}_{k}^{(p)}\right|\right) \Theta\left(r_{p}-\left|\mathbf{r}_{i}-\mathbf{r}_{k}^{(p)}\right|\right) \hat{\mathbf{r}}_{i k}^{(p)}$, where $\mathbf{r}_{k}^{(p)}$ is the location of pin $k, \Theta$ is the Heaviside step function, $\hat{\mathbf{r}}_{i j}=\left(\mathbf{r}_{i}-\mathbf{r}_{j}\right) /\left|\mathbf{r}_{i}-\mathbf{r}_{j}\right|$ and $\hat{\mathbf{r}}_{i k}^{(p)}=\left(\mathbf{r}_{i}-\mathbf{r}_{k}^{(p)}\right) /\left|\mathbf{r}_{i}-\mathbf{r}_{k}^{(p)}\right|$. The pin density is $n_{p}=1.0 / \lambda^{2}$ and the pinning force is $f_{p}=1.5 f_{0}$. The Lorentz force from an applied current $\mathbf{J}=J \hat{\mathbf{y}}$ is modeled as a uniform driving force $\mathbf{f}_{d}$ on the vortices in the $x$-direction. We initialize the vortex positions by performing simulated annealing with $f_{d} / f_{0}=0.0$. We then gradually increase $f_{d}$ to its final value by repeatedly increasing $f_{d}$ by $0.004 f_{0}$ and remaining at each drive for $10^{4}$ time steps, where $d t=0.02$. If we increase the drive more rapidly than this, the reordered vortex lattice that forms at higher drives may fail to align its principle axis in the direction of the driving. Slow increases in $f_{d}$ always produce an aligned lattice. Once the final $f_{d}$ value is reached we equilibrate the system for an additional $2 \times 10^{4}$ steps and then begin applying a force in the transverse direction $f_{d}^{y}$ which we increase by $0.0001 f_{0}$ every $10^{4}$ time steps. We monitor the transverse velocities $V_{y}=\left(1 / N_{v}\right) \sum_{i=1}^{N_{v}} \mathbf{v}_{i} \cdot \hat{\mathbf{y}}$ to identify the transverse critical current.

In Fig. 1 we show $V_{y}$ versus the transverse drive $f_{d}^{y}$ at longitudinal drives of $f_{d} / f_{0}=1.0$ and $f_{d} / f_{0}=3.0$ for a system with a longitudinal depinning threshold of $f_{c}^{x} / f_{0} \approx 0.5$. For $f_{d} / f_{0}=3.0$ the vortex lattice is free of defects and the vortices move in well defined 1D channels as seen from the vortex trajectories in the right inset, in agreement with previous simulations [13]. In this case there is clear evidence for a transverse barrier with $f_{c}^{y} / f_{c}^{x} \approx 0.01$, approximately 100 times smaller than the longitudinal depinning threshold, in agreement with earlier simulations [10,11]. Thus, the vortex lattice resists changing its direction of motion. For $f_{d} / f_{0}=1.0$, the vortex lattice is highly defected and the $1 \mathrm{D}$ channel structure is lost (as seen in the left inset of Fig. 1). In this case the transverse barrier is absent since the lattice has no particular alignment and can readily change the direction of its motion. In the absence of pinning, $f_{c}^{y}=0$ for all drives $f_{d}$, as indicated by the top curve in Fig. 1.

We ran a series of simulations in which the final longitudinal drive $f_{d}$ was varied in order to determine the dependence of the magnitude of the transverse barrier on the magnitude of the longitudinal drive, as well as on the density of defects in the vortex lattice. In Fig. 2 we plot the resulting transverse depinning thresholds $f_{c}^{y}$ and the fraction of six-fold coordinated vortices $P_{6}$ (calculated from the Voronoi or Wigner-Seitz cell construction) as a function of $f_{d}$. For longitudinal drives $f_{d} / f_{0}>1.5$, there are no defects in the vortex lattice (indicated by the fact that $P_{6} \approx 1.0$ ) and $f_{c}^{y}$ is roughly constant, $f_{c}^{y} / f_{c}^{x} \approx 0.01$. Below $f_{d} / f_{0} \lesssim 1.5$ defects begin to appear in the vortex lattice as adjacent moving channels decouple, and the overall vortex lattice develops a moving smectic structure. [13]. The transverse critical current $f_{c}^{y}$, which is still finite in this phase, becomes progressively reduced as more defects are generated. At the lowest drives, $f_{d} / f_{0} \gtrsim 1.0$, the transverse critical force is lost when the $1 \mathrm{D}$ channels are completely destroyed and the vortex lattice enters the amorphous plastic flow phase shown in the left inset of Fig. 1. The dislocations in the lattice, which were aligned perpendicular to the vortex motion at $f_{d} / f_{0}>1.0$, become randomly aligned at the transition to plastic flow. The loss of $f_{c}^{y}$ thus coincides with the loss of alignment of the defects and the destruction of the $1 \mathrm{D}$ channels.

We have also checked the effect of finite system size on the magnitude of the transverse critical force for $f_{d} / f_{0}=$ 1.5, 2.0 and 3.0 for different system sizes $(L=24 \lambda, 36 \lambda$, $48 \lambda$ and $60 \lambda)$. In the inset of Fig. 2 , we show that $f_{c}^{y} / f_{c}^{x}$ is not affected by the system size. These results support the idea that it is the presence of defects in the lattice that reduce or destroy the transverse barrier, rather than any type of matching effect with the system size, and that as long as some form of channeling occurs the barrier will still be present.

In order to view the dynamics of the vortex lattice at the transverse depinning threshold we plot in Fig. 3 the vortex positions and trajectories for the same system shown in Fig. 1 with $f_{d} / f_{0}=3.0$ and $f_{d}^{y} / f_{c}^{x}=0.011$. For clarity we have highlighted a particular row of vortices. In Fig. 3(a) the principle axis of the vortex lattice is aligned with the direction of the drive and the ordered lattice is moving along this axis. In Fig. 3(b) the entire vortex lattice has translated by one lattice constant in the transverse direction. During the transition the lattice moves at an angle to the longitudinal drive, following a different axis of the lattice. Once the vortices have moved one lattice constant transverse to the drive, they begin moving along the same channels that formed before the transverse translation. After this, the vortices move along the longitudinal direction once again, as in Fig. 3(c), before jumping by another lattice constant in the transverse direction. At the transverse depinning transition, the vortex lattice thus moves in a staircase like manner, always keeping its principle axis aligned in the direction of the original longitudinal drive and always translating along one of the axes of the lattice. As $f_{c}^{y}$ is increased the frequency of jumps in the transverse direction increases. If $f_{c}^{y}$ is increased to a high enough value, we have found evidence that the vortex lattice will reorient itself with the net driving force via the creation of a grain boundary. This will be discussed in more detail elsewhere. The transverse depinning transition is unlike the longitudinal depinning transition in that the latter occurs through plastic deformations of the lattice and 
the generation of a large number of defects. In contrast, the transverse depinning transition is elastic.

The fact that the vortices move periodically by a lattice constant in the transverse direction is a result of the fact that the longitudinal channels followed by the vortices are uniquely determined by the underlying disorder [15]. The vortices jump from one of these stable channels to another, giving the same effect as a washboard potential. This periodic effect occurs only for a moving lattice in which 1D channels have formed; to a stationary lattice, the disorder would appear random.

A consequence of the staircase-like vortex motion just above the transverse depinning threshold is that the net transverse vortex velocity at a fixed $f_{d}^{y}$ should show a clear washboard frequency which should increase for increasing $f_{d}^{y}$. In Fig. 4(a) we plot $V_{y}$ for samples with $f_{d}^{y}$ held fixed at several different values just above the transverse depinning threshold for a system with $f_{d} / f_{0}=3.0$. For $f_{d}^{y} / f_{c}^{y}=0.011$ the $V_{y}$ shows periodic pulses which correspond to the correlated transverse jumps of the vortex lattice seen in Fig. 3. The flat portions of the voltage signal correspond to time periods when the lattice is moving only in the longitudinal direction, between hops. For increasing transverse drive the frequency of these pulses also increases. The additional structure in the $V_{y}$ voltage pulses at lower values of $f_{d}^{y}$ is characteristic of the underlying pinning, and varies for different disorder realizations. It occurs when the vortex lattice moves slightly unevenly, with a small wobble, but no defects or tearing occur in the lattice. The main feature of large periodic pulses is always observed, and the wobble dies away at larger transverse drives. In Fig. 4(b) we show that the Fourier transform of the velocity signal $V_{y}$ for a driving force of $f_{d}^{y} / f_{c}^{y}=0.016$ exhibits a resonance frequency at $\nu=6.0 \times 10^{-5}$ inverse MD steps. In Fig. 4(c), the resonant frequency increases linearly with $f_{d}^{y}$. We find that this resonance persists for $f_{d}^{y}$ up to ten times larger then the transverse depinning threshold. It should be possible to detect this washboard frequency with Hall-noise measurements.

In recent experiments employing an STM to directly image a slowly moving vortex lattice [21], evidence for staircase-like motion of the flux lattice has been observed. In these experiments the direction of the driving force could not be directly controlled, but was assumed to be at a slight angle with respect to the principle vortex lattice vector, so that a transverse component of the driving force was present. Further experiments in which the magnitude and direction of the drive can be directly controlled are needed; however, experimental imaging techniques such as STM or Lorentz microscopy [22] seem highly promising.

In summary we have investigated the transverse depinning of moving vortex lattices interacting with random disorder. We find that for high longitudinal drives where the vortex lattice is defect free a finite transverse bar- rier forms. For lower drives where defects in the vortex lattice form and the vortex lattice has a smectic structure the transverse barrier is reduced but still finite. In the highly disordered plastic flow phase the transverse barrier is absent. The transverse depinning transition is elastic, unlike the plastic longitudinal depinning transition, and near this transition the vortex lattice moves in a staircase-like fashion. We observe a washboard frequency in the transverse voltage signal which can be detected for transverse drives up to ten times the depinning drive.

We thank T. Giamarchi, P. Kes, P. Le Doussal, F. Nori, R. Scalettar, and G. Zimányi for helpful discussions. We acknowledge support from CLC and CULAR, administered by the University of California.

[1] S. Bhattacharya and M.J. Higgins, Phys. Rev. Lett. 70, 2617 (1993); A.C. Marley, M.J. Higgins and S. Bhattacharya, Phys. Rev. Lett. 74, 3029 (1995); M.J. Higgins and S. Bhattacharya, Physica C 257, 232 (1996).

[2] U. Yaron et al., Nature 376, 753 (1995).

[3] M.C. Hellerqvist et al., Phys. Rev. Lett. 76, 4022 (1996).

[4] W. Henderson et al., Phys. Rev. Lett. 77, 2077 (1996).

[5] A. Duarte et al., Phys. Rev. B 53, 11336 (1996); F. Pardo et al., Phys. Rev. Lett. 78, 2644 (1997).

[6] M. Marchevsky et al., Phys. Rev. Lett. 78, 531 (1997).

[7] F. Pardo et al., Nature 396, 348 (1998).

[8] A.M. Troyanovski, J. Aarts, and P.H. Kes, Nature 399, 665 (1999).

[9] A.E. Koshelev and V.M. Vinokur, Phys. Rev. Lett. 73, 3580 (1994).

[10] K. Moon, R.T. Scalettar, and G.T. Zimányi, Phys. Rev. Lett. 77, 2278 (1996).

[11] S. Ryu et al., Phys. Rev. Lett. 77, 5114 (1996).

[12] H.J. Jensen, A. Brass and A.J. Berlinsky, Phys. Rev. Lett. 60, 1676 (1988); A.-C. Shi and A.J. Berlinsky, Phys. Rev. Lett. 67, 1926 (1991); N. Grønbech-Jensen, A.R. Bishop and D. Dominguez, Phys. Rev. Lett. 76, 2985 (1996); M.C. Faleski, M.C. Marchetti, and A.A. Middleton, Phys. Rev. B 54, 12427 (1996); S. Spencer and H.J. Jensen, Phys. Rev. B 55, 8473 (1997); D. Dominguez, Phys. Rev. Lett. 82, 181 (1999).

[13] C.J. Olson, C. Reichhardt, and F. Nori, Phys. Rev. Lett. 81, 3757 (1998).

[14] A.B. Kolton, D. Dominguez, and N. Grønbech-Jensen, cond-mat/9906157.

[15] T. Giamarchi and P. Le Doussal, Phys. Rev. Lett. 76, 3408 (1996).

[16] T. Giamarchi and P. Le Doussal, Phys. Rev. B 57, 11 356 (1998).

[17] L. Balents, M.C. Marchetti, and L. Radzihovsky, Phys. Rev. B 57, 7705 (1998).

[18] S. Scheidl and V.M. Vinokur, Phys. Rev. E 57, 2574 (1998).

[19] C. Reichhardt and F. Nori, Phys. Rev. Lett. 82, 414 (1999); V.I. Marconi and D. Dominguez, Phys. Rev. Lett. 82, 4922 (1999).

[20] A. Schmid and W. Hauger, J. Low Temp. Phys. 11, 667 (1973). 
[21] P. Kes, private communication.

[22] T. Matsuda et al., Science 271, 1393 (1996).

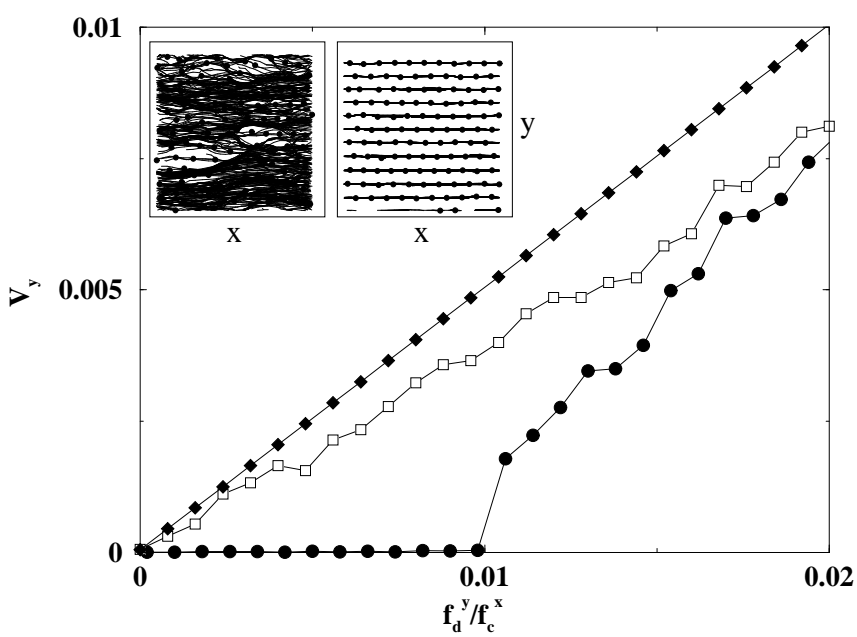

FIG. 1. Transverse voltage $V_{y}$ versus transverse driving force $f_{d}^{y}$. Diamonds: A sample with no pinning, $f_{p} / f_{0}=0$, shows no transverse barrier at any drive (here, $\left.f_{d} / f_{0}=3.0\right)$. Squares and circles: a sample with pinning strength $f_{p} / f_{0}=1.5$ and longitudinal critical current $f_{c}^{x} / f_{0}=0.5$. Squares: For a longitudinal driving force $f_{d} / f_{0}=1.0$ the vortices flow plastically (left inset) and there is no transverse barrier. Circles: For a longitudinal driving force $f_{d} / f_{0}=3.0$ the vortices flow in well-defined channels (right inset) and a transverse barrier of $f_{c}^{y} / f_{c}^{x}=0.01$ appears. Insets: Filled circles represent vortices and lines show the paths traveled by the vortices while moving over randomly spaced pinning sites (not shown). Left inset: plastic flow; right inset: channel flow.

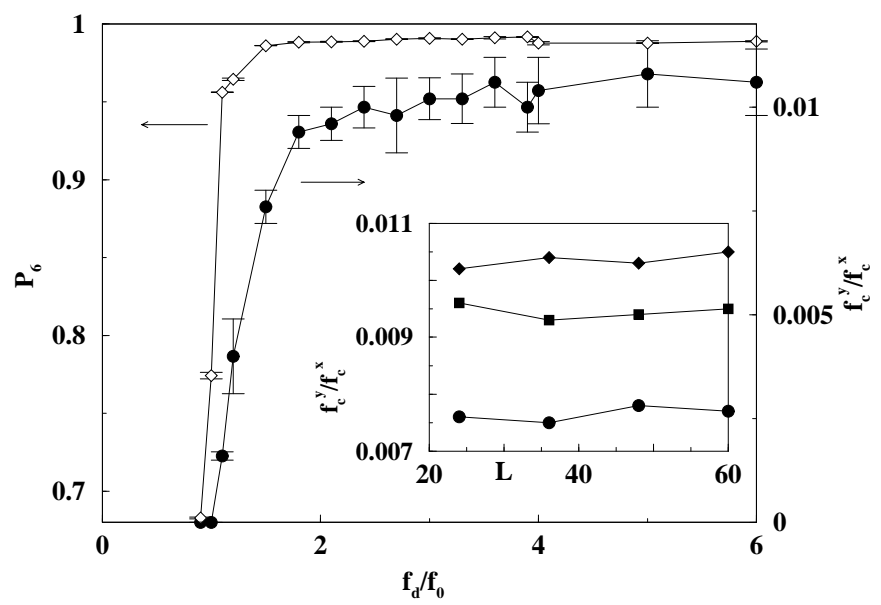

FIG. 2. Circles: Transverse critical force $f_{c}^{y} / f_{c}^{x}$ versus longitudinal driving force $f_{d}$. Diamonds: Corresponding fraction of six-fold coordinated vortices, $P_{6}$. The transverse critical force saturates for a reordered vortex lattice, $f_{d} / f_{0}>1.8$, but drops as the lattice becomes defected, reaching zero at $f_{d} / f_{0} \approx 1.0$. Inset: Transverse critical force for different system sizes $L=24 \lambda, 36 \lambda, 48 \lambda$, and $60 \lambda$. Circles: $f_{d} / f_{0}=1.5$; Squares: $f_{d} / f_{0}=2.0$; Diamonds: $f_{d} / f_{0}=3.0$.

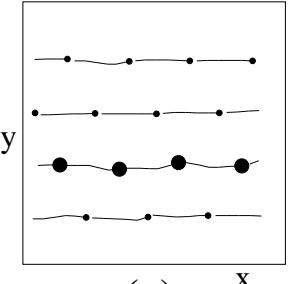

(a)

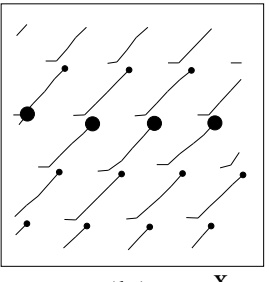

(b)

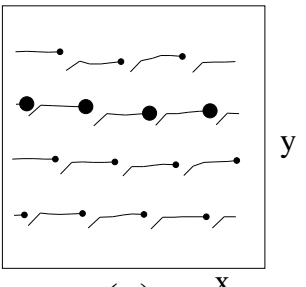

(c)
FIG. 3. Consecutive simulation images of vortex motion just above the transverse critical force, $f_{d}^{y} / f_{c}^{y}=0.011$. Circles represent vortices and lines indicate paths followed by the vortices. A particular row of vortices has been highlighted. In (a) the vortices are moving in the direction of the applied longitudinal drive. In (b) the vortex lattice changes its direction of motion and follows a different lattice vector until it has translated by one lattice constant. In (c) the lattice again switches direction and continues to flow in the longitudinal channels. The frequency at which the vortex lattice hops from channel to channel increases with increasing transverse drive.

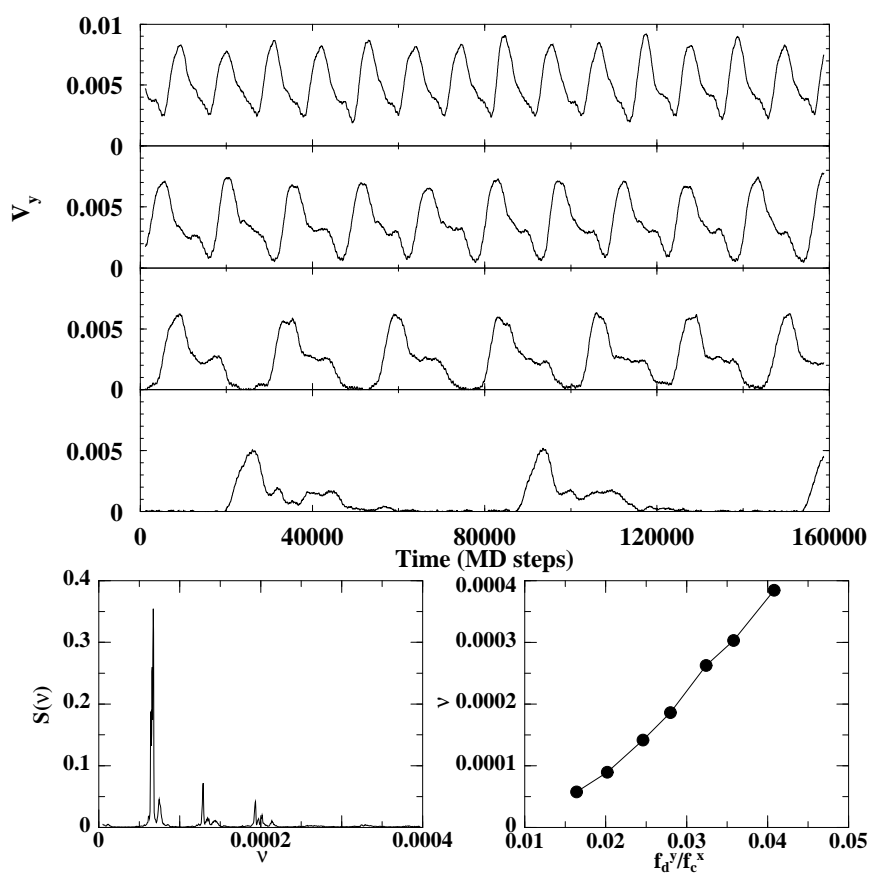

FIG. 4. (a) Transverse voltage signal $V_{y}$ as a function of time for a sample with longitudinal drive $f_{d} / f_{0}=3.0$ at four different transverse driving currents $f_{d}^{y}$. Bottom to top: $f_{d}^{y} / f_{c}^{x}=0.011, f_{d}^{y} / f_{c}^{x}=0.014, f_{d}^{y} / f_{c}^{x}=0.016$, and $f_{d}^{y} / f_{c}^{x}=0.20$. Each pulse corresponds to the vortex lattice moving one lattice constant in the transverse direction. (b) Power spectrum $S(\nu)$ of the transverse voltage noise signal $V_{y}$ for a sample with $f_{d} / f_{0}=3.0$ and $f_{d}^{y} / f_{c}^{x}=0.016$. A clear narrow band signature appears at $\nu=6.0 \times 10^{-5}$. (c) Location of the narrow band peak $\nu$ for different transverse driving forces $f_{d}^{y}$. 